\title{
Enhanced Spectrum Utilization for Existing Cellular Technologies Based on Genetic Algorithm in Preview of Cognitive Radio
}

\author{
K. SRIDHARA ${ }^{1}$, Aritra NAYAK ${ }^{2}$, Vikas SINGH $^{2}$, P. K. DALELA ${ }^{2}$ \\ ${ }^{1}$ Member Technology, Government of India, New Delhi, India \\ ${ }^{2} \mathrm{C}-\mathrm{DOT}$, New Delhi, India \\ E-mail:pdalela@gmail.com \\ Received September 2, 2009; revised October 21, 2009; accepted November 29, 2009
}

\begin{abstract}
This paper attempts to find out the distributed server-based dynamic spectrum allocation (DSA) within liberalized spectrum sharing regulation concept as an alternative to existing regulation based on fixed frequency spectrum allocation schemes towards development of cognitive radio for coverage-based analogy. The present study investigates a scenario where a block of spectrum is shared among four different kinds of exemplary air interface standards i.e., GSM, CDMA, UMTS and WiMAX. It is assumed to offer traffic in an equally likely manner, which occupy four different sizes of channel bandwidths for different air interfaces from a common pooled spectrum. Four different approaches for spectrum pooling at the instance of spectrum crunch in the designated block are considered, viz. channel occupancy through random search, existing regulation based on fixed spectrum allocation (FSA), FSA random and channel occupancy through Genetic Algorithm (GA) based optimized mechanism to achieve desired grade of service (GoS). The comparisons of all the approaches are presented in this paper for different air interfaces which shows up to $55 \%$ improvement in GoS for all types of air interfaces with GA-based approach in comparison to existing regulations.
\end{abstract}

Keywords: DSA, GA, GSM, CDMA, UMTS, WiMAX, Cognitive Radio

\section{Introduction}

The sophistication possible in a software-defined radio (SDR) [1-3] has now reached the level where each radio can conceivably perform beneficial tasks that help the user, network, and minimize spectral congestion. Radios are already demonstrating one or more of these capabilities in limited ways [4,5]. A simple example is the adaptive digital European cordless telephone (DECT) wireless phone, which finds and uses a frequency within its allowed plan with the least noise and interference on that channel and time slot [6]. Of these capabilities, conservation of spectrum is already a national priority in international regulatory planning. As on date, there are certain rules [4] by which a fixed spectrum is allocated to designated technology, and other technology/service provider cannot use this spectrum. We are interested to investigate this hypothesis in the case of cognitive radio $[5,6]$ i.e., in case of availability of spectrum anywhere, any technology/service provider user can use that to accommodate maximum subscribers within limited spectrum.
As an example of the potential for utilizing the time varying nature of the traffic, we consider four different radio networks: GSM, CDMA, UMTS and WiMAX. We also assume that these radio networks might be used to support different services, e.g. voice telephony on GSM [7], CDMA, broadband internet access along with video streaming on UMTS (for individual subscribers) and WiMAX (mainly for corporate connections). The traffic pattern (and therefore demand for frequency spectrum) seen on each of these networks would vary throughout the day. Example traffic patterns are shown in Figure 1 based on the assumption that voice telephony and corporate connection demands will be high during office time while individual broadband internet subscriber demand will be high before and after office hours. Here GSM traffic variation has been drawn with the help of reference [7] whereas the traffic variation of CDMA, UMTS and WiMAX are drawn based on above assumption.

Here we assumed that a block of spectrum is shared among four different kinds of exemplary air interface standards i.e., GSM, CDMA, UMTS and WiMAX which 


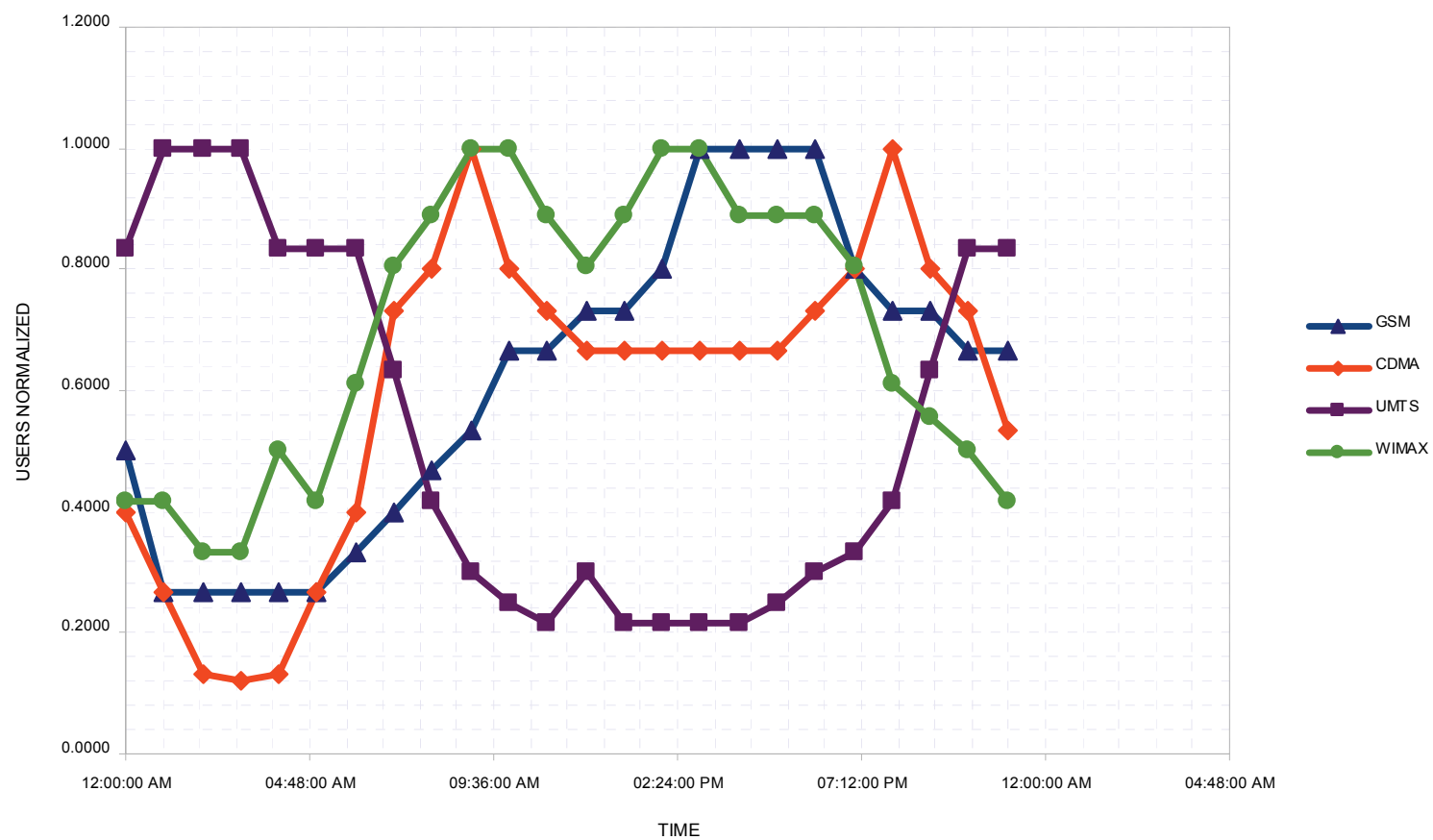

Figure 1. The peak traffic variations of the four types of technologies that share the spectrum over a 24-hour period.

occupies $200 \mathrm{kHz}, 1.25 \mathrm{MHz}, 5 \mathrm{MHz}$ and $10 \mathrm{MHz}$ frequency bandwidths respectively. The relation among different technologies is spectrum occupancy related for coverage-based analogy that in case of secondary users, i.e., if UMTS allocated spectrum is fully occupied and UMTS subscriber (which will be secondary user for other technology spectrum) wants to grab channel in some other allocated technology spectrum e.g. GSM, then it would also require full $5 \mathrm{MHZ}$ instead of $200 \mathrm{KHz}$ for GSM. The distributed server instead of centralized server-based approach has been taken to reduce computing time. The fixed frequency spectrum has been allocated to these different technologies. Initially, some traffic patterns based on actual traffic load have been assumed for all these four technologies during a day. As per present regulations, initially as traffic (number of users) increases, the specific technology user tries to grab channel within its allocated frequency spectrum slot and in case of unavailability of frequency resources user would be dropped. The fixed spectrum allocation (FSA) does have some disadvantages. For example, most communication networks are designed to cope with a certain maximum amount of traffic. The dimensioning of the network is based on the "busy hour", which is the time of the peak use of the network. If this network uses its allocated spectrum fully during this hour, then the rest of the time the spectrum is not fully utilized. A similar pattern is also seen with other services, hence, with the help of dynamic spectrum allocation the dropped users can be reduced and hence GoS can be enhanced. This paper leads through the technologies and regulatory considerations to support spectrum management and optimizations that raise SDR's capabilities and make it a cognitive radio. Many technologies have come together to result in the spectrum efficiency and cognitive radio technologies may be considered as an application on top of a basic SDR platform. In the present paper, biologically inspired Genetic Algorithm (GA) [8-10] based dynamic spectrum access (DSA) $[4,11,12]$, with distributed server based approach [13], as one of its intended applications have been proposed to reduce blocked users i.e., GoS by utilizing unutilized spectrum. This paper is organized as follows. In Section 2, simulation model for GoS of existing regulation based on FSA and other approaches which includes GA-based optimized mechanism of channel grabbing has been explained along with traffic model. In Section 3, a brief review to GA and its applicability in simulation has been explained. In Section 4, simulation results are shown and Section 5 concludes this study.

\section{Simulation Model}

In this section, the concepts behind simulation of GoS with time for 4 different scenarios i.e. Fixed Spectrum Allocation (FSA), FSA random (FSA RAND), total random (TOT_RAND) and GA optimized mechanism have been elaborated.

The random spectrum allocation situation is analogous to road traffic control for multiple lanes dedicated to a 
particular type of vehicle philosophy i.e., where allocation of frequency spectrum is fixed for each technology within a certain frequency range. The different sizes of vehicles can be compared to different channel bandwidth requirements for the different air interface standards. A deregulated regime is analogous to having a traffic circle at the road junction wherein every vehicle finds a suitable slot proportional to its size in the circle; the circle itself represents the available spectrum pool. It is assumed that a pool of $\mathrm{F}=120 \mathrm{MHz}$ of spectrum is available for four different bandwidths, viz. $\mathrm{B} 1=0.2 \mathrm{MHz}, \mathrm{B} 2=1.25 \mathrm{MHz}$, $\mathrm{B} 3=5 \mathrm{MHz}$ and $\mathrm{B} 4=10 \mathrm{MHz}$, all operating in Time Division Duplex mode, i.e., pairing of frequencies for uplink and downlink is not considered. The entire band of $120 \mathrm{MHz}$ is quantized in steps of $\mathrm{f}=0.05 \mathrm{MHz}$ for simulation purposes.

The above analogy is repeated with fixing slots of frequency spectrum for different technologies. At the time of congestion pertaining to one technology, the additional amount of required frequency spectrum can be borrowed from other technology slots if they have spare frequency spectrum at that moment. The optimization of bandwidth borrowing and lending is proposed by GA i.e., introducing regulations based on DSA with GA. Four different approaches for spectrum pooling at the instance of spectrum crunch in the designated block are considered. In first approach, channel occupancy through random search in complete pooled frequency spectrum is simulated. This is done by allocating chunks of the quantized spectrum to the various users of the four technologies. For example, a GSM user gets four blocks of $50 \mathrm{KHz}$ i.e., $200 \mathrm{KHz}$ for a call but this allocation is done on the basis of a random channel grabbing where the channel to be grabbed is generated by a random generator. In second approach, the channel occupancy through existing regulations based on fixed spectrum allocation (FSA) is simulated. This is done by allocating users in their fixed spectrums one after the other is a sequential manner until space runs out for new users on which we simply do a sequential scan to find if there is any empty space to accommodate the new user else the call is dropped. In third approach, FSA random i.e., allocation of resources to different technologies in the designated slots only through randomized search is simulated. For this scheme of allocation, the users are allocated space only in their respective spectrums just like FSA the difference being that the users grab channels within the spectrums allocated with the help of a randomly generated channel number. Lastly the channel occupancy in designated slots through Genetic Algorithm (GA) based optimized mechanism is simulated to achieve the desired grade of service (GoS). The scheme of which will be explained in Section 3. The comparisons of all the four approaches for individual and combined traffic are presented in Section 4.

Traffic Model: For the simulation purpose we assume a perfect channel (either idle or busy). Poisson random process [14] is used to model the arrival traffic with rate $\lambda$ and the inter arrival time is negative-exponentially dis- tributed with mean $1 / \lambda$. The hold time duration is generated from Gaussian random process [14] which is negative-exponentially distributed with mean $1 / \mu$. It is assumed that there is some time spent for spectral scanning and this is required to be less than the inter arrival rate.

\section{A Brief Review to GA and its Applicability in Simulation}

A Genetic Algorithm (GA) [10] is a search algorithm based on the principles of evolution and natural genetics. It combines the exploitation of past results with the exploration of new areas of the search space. By using survival of the fittest techniques combined with a structured yet randomized information exchange, a GA [10] can mimic some of the innovative flair of human search.

In our case, the GA [10] is used to maximize the spectral utilization by using the least bandwidths to create spectrum opportunities for competing users in the spectrum. We can describe GA [10] to find solution for blocked users as follows. For example, in a network with $d$ users, the number of different ways ( $\Gamma$ ) [15] these users can be allocated to a spectrum bandwidth (assuming reuse factor $=1$ ) without repetition can be computed using the following equation.

$$
\begin{gathered}
\Gamma=\sum_{r=1}^{d}\left(\begin{array}{l}
d \\
r
\end{array}\right) \\
\left(\begin{array}{c}
d \\
r
\end{array}\right)=\frac{d !}{r !(d-r) !}
\end{gathered}
$$

This would lead to a total of $\boldsymbol{r}^{\mathrm{m}}$ [15] different combinations for the $m$ spectrum bands. For instance, a system with blocked users $d=15$ and four spectrum bands for different technologies $(m=4)$ would have approximately $2^{56}$ possible allocations and to find the optimal solution, exhausting all combinations would not be efficient, as a processor checking one billion solutions per second requires approximately 2.3 years to analyze all permutations. Therefore analytical methods may not be suitable for such type of problems. Thus, GA [10] has been successfully applied to this class of combinatorial optimization problems.

Simplicity of operation and power of effect are two main attractions of the GA [10] approach. The effectiveness of the GA [10] depends upon an appropriate mix of exploration and exploitation. Three operators to achieve this are selection, crossover, and mutation [10]. GA has parameters and variables to control the algorithm. There are evolution operation, genetic operations and parameter settings in GA. First evolution operation is selection. Typical methods for selection [10] are encoding scheme, fitness function and seeding. Second genetic operations mainly have crossover and mutation operations. The selection parameters are defined as follows: 
1) Encoding Scheme: Encoding scheme [10] is one of the important and crucial aspects to control the performance of Genetic Algorithm [10]. It refers to the method of mapping the problem parameters into a chromosome [10], which decides the nature of being a weak or strong coding. Encoding scheme can be strong or week in terms of its capability to explore the search space and strong encoding scheme exploits more features of the solution domain.

The encoding method is a global approach to the problem. It is global because any chromosome has enough information to describe a set of channel- borrowings for the entire network.

A chromosome is composed in the following way. For every slot of technology in the spectrum, there is a major chromosome slot or super-gene. Within each super-gene, there are four actual genes. These genes represent the four technologies within a spectrum [8].

A Gene is an array of length 10. At the first location of the array, we keep the number of blocked slots and second location has the number of free slots which were formed by quantization of the spectrum. Next four locations contain the data about number of slots borrowed from other technologies and last four locations contain information about slots lent to other technologies. Thus the super-gene is formed as matrix of order 4 X 10 where each row of the gene represents one particular technology.

\section{Gene Structure}

$\begin{array}{llllllllll}1 & 2 & 3 & 4 & 5 & 6 & 7 & 8 & 9 & 10\end{array}$

The allocated spectrum for different technologies in the present study is in the ratio of 1:2:4:5 for GSM, CDMA, UMTS, and WiMAX respectively. However, to show results independent from allocated frequency, spectrum slots for individual technologies GoS has been evaluated.

2) Fitness Function: The fitness function [10] is measuring mechanism to rank the quality of a chromosome. It serves the only link between the problem and algorithm to search the optimal solutions.

$$
\begin{gathered}
\text { fitniess }=\sum \alpha * \text { accommodated }+ \\
\sum \beta * \text { unserviced }+ \\
\sum \mu *(\text { expected congestion } * \text { serviced })
\end{gathered}
$$

where $\alpha, \beta, \mu$ are constant values to suit the environment; such that, $\alpha \varepsilon\{\mathrm{w} 1, \mathrm{w} 2, \mathrm{w} 3\}$ and $\mathrm{w} 1>\mathrm{w} 2>\mathrm{w} 3 ; \beta<0$ and $\mu<0$ for all cases.

accommodated is the measure of whether the given number of users can be serviced by the free space and depending upon this $\alpha$ takes values from w1, w2 and w3.

unserviced is the number of users blocked during the congestion and expected congestion is calculated by working out possibly how much traffic is going to arrive in each band and finding a ratio to this expected traffic and the maximum capacity of the band.

3) Seeding: We have presented the solution having initial gene to zero which is the first step of seeding [10].
While with channel-borrowing heuristic, the initial gene can be stated with one of the possible solutions and then the genetic flow is operated to get the best solution. Definitely with the later approach, the numbers of iterations to converge to a solution are decreased. This approach is applied with the improved pluck operation [9].

The genetic operations are defined as follows:

1) Crossover: Crossover [10] is the most important function in GA [10], which produces children as new chromosomes from the process of combining two chromosomes (parents). The operation of crossover may gives the children (new chromosomes) with better fitness as it takes best attributes from both the parents [10] we have used single point crossover as shown below.

First matrix | cut point

1234567895

2343487358

5675643149

9937261532

Second matrix

$$
\text { | cut point }
$$

4565746579

4358794798

$1226 \quad 634893$

5393766281

The offspring or children [10] generated from two parental matrices are:

$$
\begin{aligned}
& \text { offspring } 1 \\
& 45656765059 \\
& 435587488358 \\
& 12266344149 \\
& 539362253
\end{aligned}
$$

offspring 2

$$
\begin{aligned}
& 1234546779 \\
& 234349879 \\
& 56756438 \\
& 99377266681
\end{aligned}
$$

This crossover function facilitates not to copy entire second half of the second matrix to the first matrix, while it retains the common elements of the parental matrix, which is essential for borrowing policy to preserve necessary information or the parent characteristics of the children. However if row wise second half of the parental matrices are entirely different then this crossover function serves as normal crossover function discussed initially.

2) Mutation: The mutation [10] operator is responsible for diversity into a population. In the first phase of results, we are using the normal mutation operator with high probability to provide diversity in genes as we have started with initial gene zero in seeding. However, before swapping the child to next population, we check the 
needy technologies that require borrowing with correct mutations applied by means of a simple probability.

\subsection{GA Application in the Simulation}

In this simulation, whenever the users find that their respective allocated spectrum are fully occupied, then they try to grab empty spectrum in the other allocated technology spectrum bands. This is where GA is employed to select an empty space among the many available by repeated iterations based on crossovers and seeding so that due to the borrowing of spectrum by another technology user i.e., the secondary users [6] quality of service (QoS) of primary users [6] are not affected. The allocation is done by first randomly seeding the population with all possible solutions and then applying fitness function to select only the healthy children [10] or fit solutions. The crossover and mutation is applied to further consolidate the results and possible scheme of allocation of unutilized spectrum based on the fitness function mentioned earlier, which keeps in mind the possible traffic pattern and future trends to evolve an optimal solution. Also before the application of GA, it is checked if a specific technology spectrum which has been full and cannot accommodate the pri- mary users, a spectrum search scanning [6] has been performed to find out the secondary users residing in this specific spectrum slot and terminates them to create space for the primary users before the application of GA. The termination is done on the basis of least number of calls to be terminated and in order of time of residence in the spectrum.

The flowchart shown in Figure 2 describes the steps employed to obtain the optimal solutions using GA [10] for DSA by using its basic operators. As shown in the flowchart, the results are obtained by following a number of iterations which is fixed as a constant named MAX ITERATION. In each of the iterations, all the basic steps are followed until we obtain an optimal solution.

\section{Results}

This section shows the comparison of GoS among fixed frequency spectrum allocation (FSA) which is existing regulation, FSA random (FSA_RAND), total randomized allocation (TOT_RAND) and GA-based DSA scheme which is proposed regulation for GSM, CDMA, UMTS and WiMAX along with mixed traffic of all these technologies.

Figure 3 shows the case of GoS of GSM for $120 \mathrm{MHz}$ common spectrum for all the technologies during a span

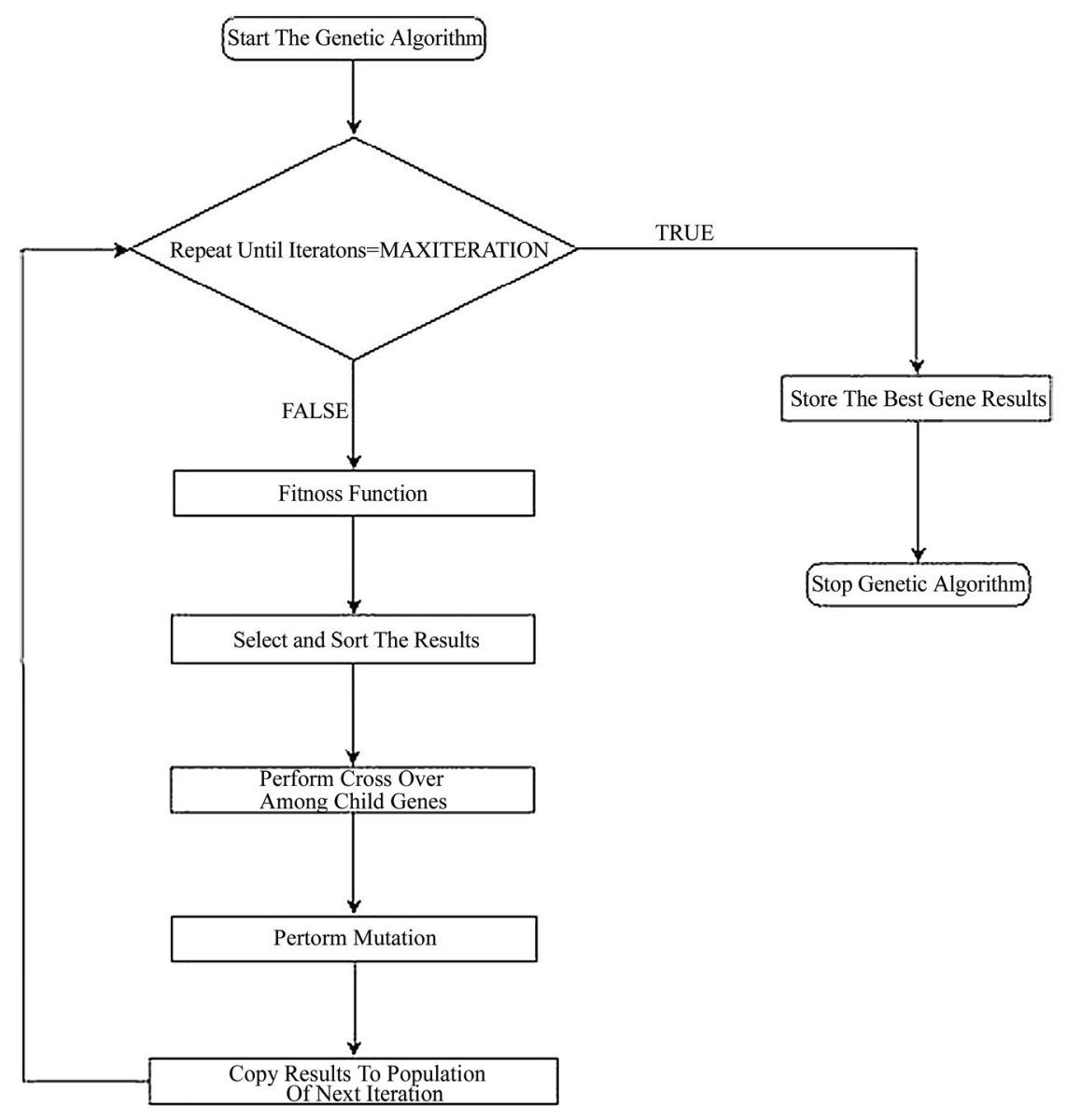

Figure 2. Flow chart of GA applicable to DSA in the present case. 
GSM BLOCKING

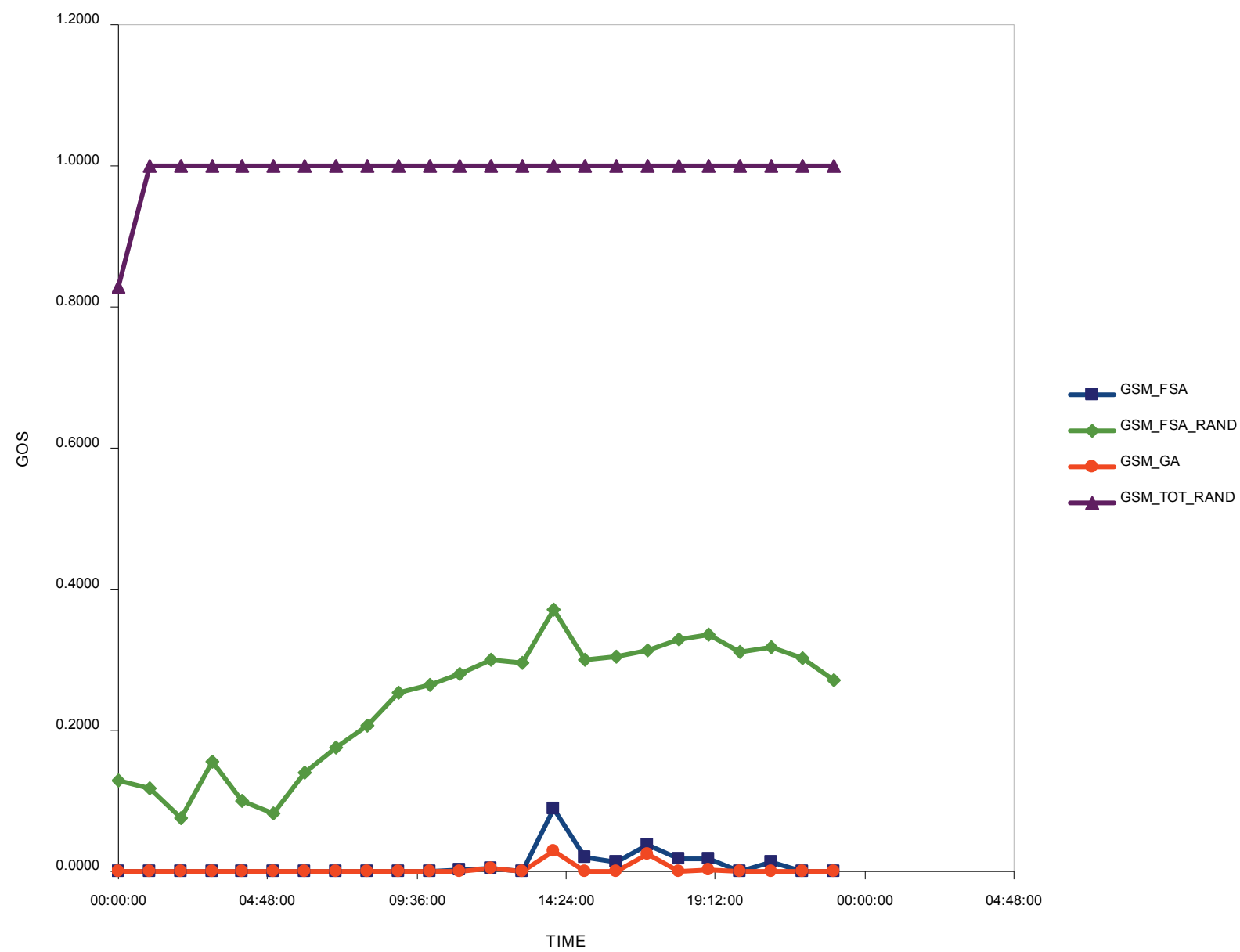

Figure 3. Comparison of GoS for GSM (0.2 MHz) users among FSA, FSA_RAND, GA-based DSA scheme and TOT_RAND over duration of 24 hours of the day.

of one day. The GSM_FSA graph shows GoS for GSM when different technologies users grab the channel slots in the allocated frequency spectrum slots only by using FSA which is existing regulation. The GSM_TOT_RAND graph shows the GoS for GSM user when there is no regulation and any technology user can grab the channel at random position if it is available. The GSM FSA RAND graph is of the randomized channel grabbing FSA where the spectrum is divided among the various technologies but the channel grab is at any random position provided it is vacant. The GSM_GA graph shows the GoS for GSM user with GA optimized DSA algorithm which is proposed regulation. It is seen that GA optimized DSA algorithm is always better than all other cases. Also it has approximately up to $72 \%$ improvement in comparison to FSA which is the best performing algorithm amongst the three. If the mean GoS is taken for the given time then a maximum of $67 \%$ improvement is noticed neglecting the cases where DSA gives zero blocking.
Figure 4 shows the case of GoS of CDMA for 120 $\mathrm{MHz}$ common spectrum for all the technologies during a span of one day. The CDMA_FSA graph shows GoS for CDMA when different technologies users grab the channel slots in the allocated frequency spectrum slots only by using FSA which is existing regulation. The CDMA_TOT RAND graph shows the GoS for CDMA user when there is no regulation and any technology user can grab the channel at random position if it is available. The CDMA FSA RAND graph is of the randomized channel grabbing FSA where the spectrum is divided among the various technologies but the channel grab is at any random position provided it is vacant. The CDMA_GA graph shows the GoS for CDMA user with GA optimized DSA algorithm which is proposed regulation. It is seen that GA optimized DSA algorithm is always better than all other cases. Also it has approximately up to $72 \%$ improvement in comparison with FSA which is the best performing algorithm amongst the three. If the mean GoS is taken for the 
given time then a maximum of $95 \%$ improvement is noticed neglecting the cases where DSA gives zero blocking.

Figure 5 shows the case of GoS of UMTS for $120 \mathrm{MHz}$ common spectrum for all the technologies during a span of one day. The UMTS FSA graph shows GoS for UMTS when different technologies users grab the channel slots in the allocated frequency spectrum slots only by using FSA which is existing regulation. The UMTS_TOT_RAND graph shows the GoS for UMTS user when there is no regulation and any technology user can grab the channel at random position if it is available. The UMTS FSA RAND graph is of the randomized channel grabbing FSA where the spectrum is divided among the various technologies but the channel grab is at any random position provided it is vacant. The UMTS_GA graph shows the GoS for UMTS user with GA optimized DSA algorithm which is proposed regulation. It is seen that GA optimized DSA algorithm is always better than all other cases. Also it has approximately up to $30 \%$ improvement in comparison to FSA which is the best performing algorithm amongst the three. If the mean GoS is taken for the given time then a maximum of $60 \%$ improvement is noticed neglecting the cases where DSA gives zero blocking.

Figure 6 shows the case of GoS of WiMAX for 120
$\mathrm{MHz}$ common spectrum for all the technologies during a span of one day. The WiMAX_FSA graph shows GoS for UMTS when different technologies users grab the channel slots in the allocated frequency spectrum slots only by using FSA which is existing regulation. The WiMAX TOT RAND graph shows the GoS for WiMAX user when there is no regulation and any technology user can grab the channel at random position if it is available. The WiMAX_FSA_RAND graph is of the randomized channel grabbing FSA where the spectrum is divided among the various technologies but the channel grab is at any random position provided it is vacant. The WiMAX_GA graph shows the GoS for WiMAX user with GA optimized DSA algorithm which is proposed regulation. It is seen that GA optimized DSA algorithm is always better than all other cases. Also it has approximately up to $15 \%$ improvement in comparison with FSA which is the best performing algorithm amongst the three. If the mean GoS is taken for the given time then a maximum of $70 \%$ improvement is noticed neglecting the cases where DSA gives zero blocking.

Figure 7 shows the case of GoS of all four technologies for $120 \mathrm{MHz}$ common spectrum for all the technologies

CDMA BLOCKING

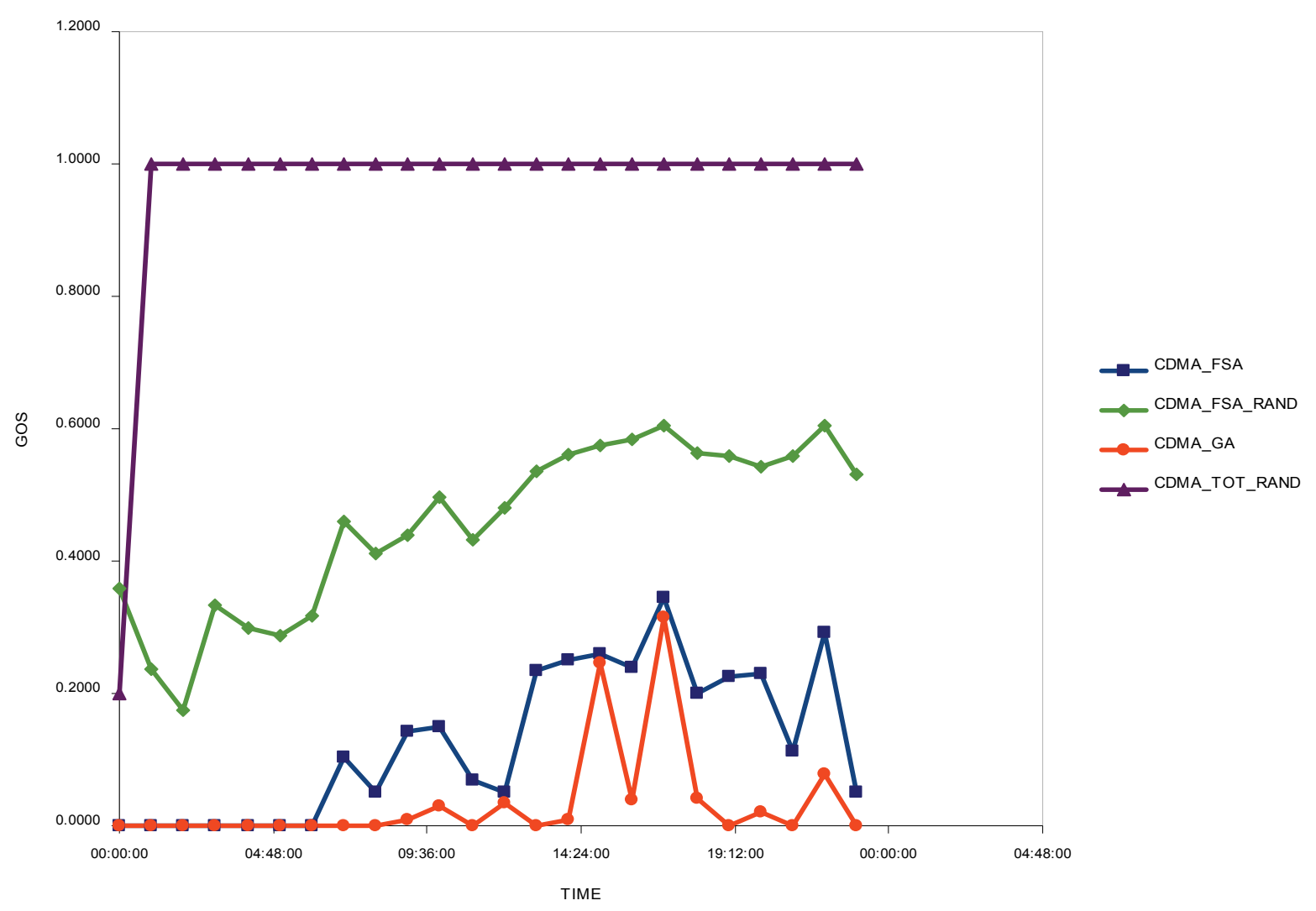

Figure 4. Comparison of GoS for CDMA (1.25 MHz) users among FSA, FSA_RAND, GA-based DSA scheme and TOT RAND over duration of 24 hours of the day. 


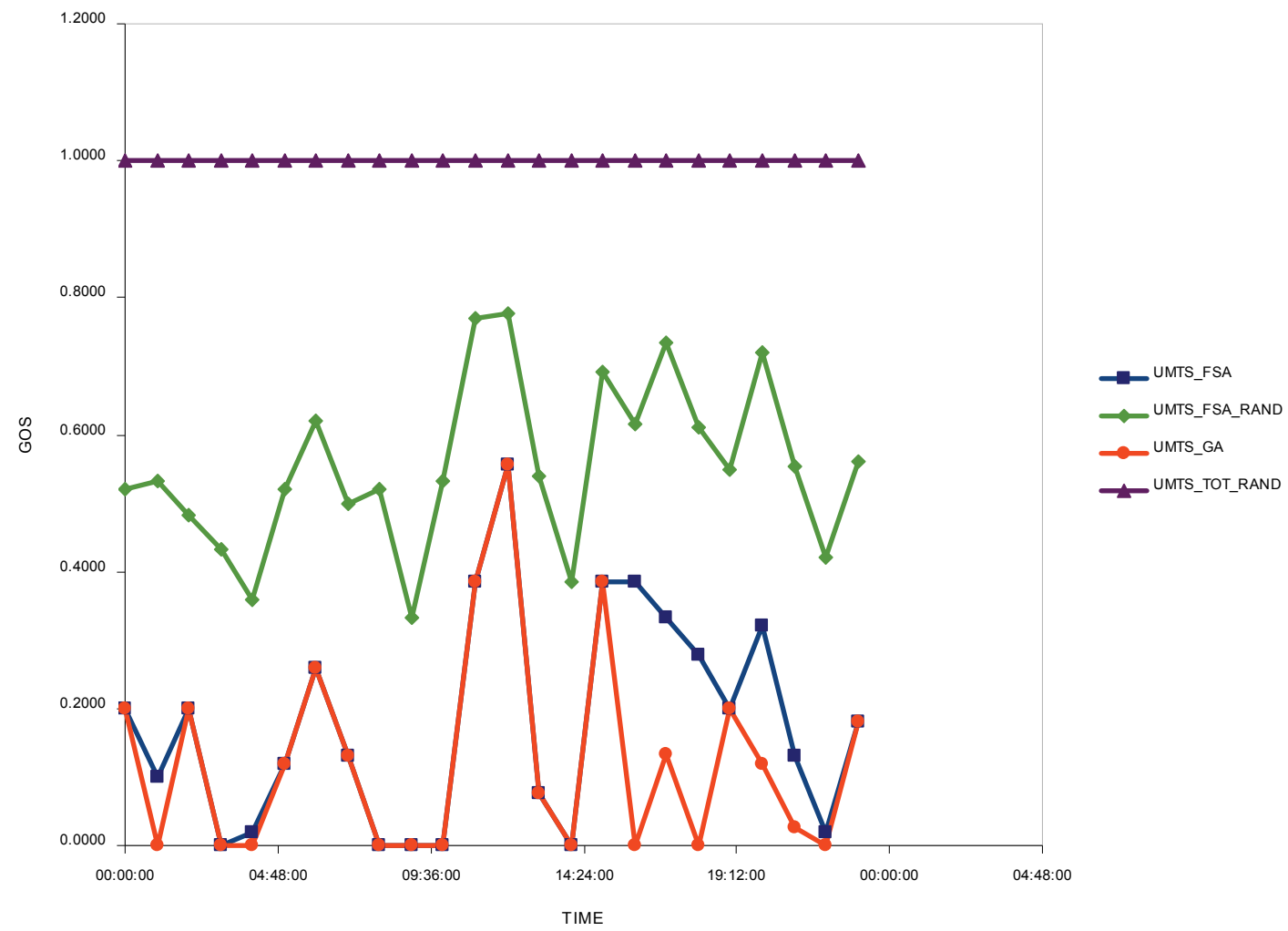

Figure 5. Comparison of GoS for UMTS (5 MHz) users among FSA, FSA_RAND, GA-based DSA scheme and TOT_RAND over duration of 24 hours of the day.

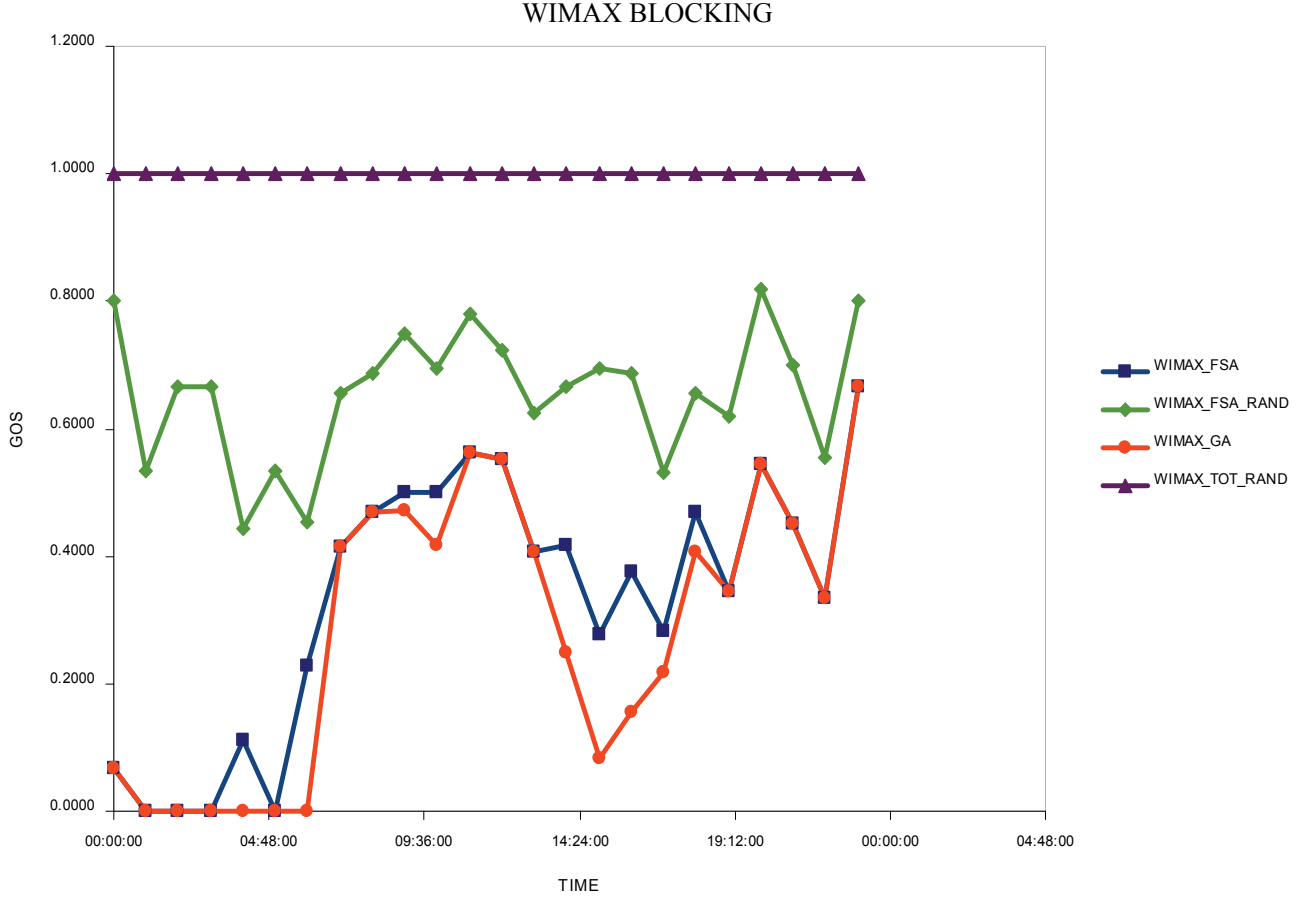

Figure 6. Comparison of GoS for WiMAX (10 MHz) users among FSA, FSA_RAND, GA-based DSA scheme and TOT_ RAND over duration of 24 hours of the day. 


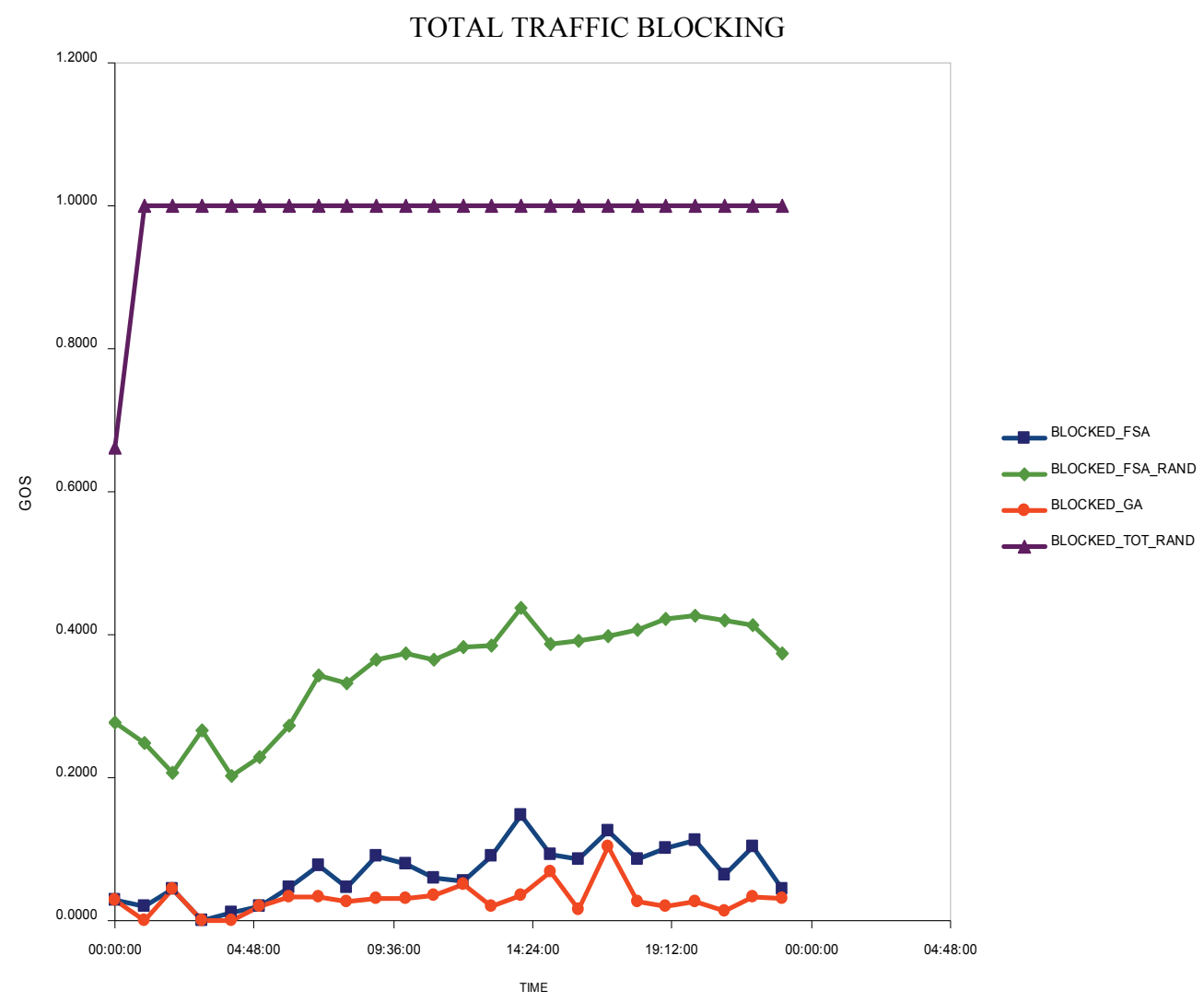

Figure 7. Comparison of GoS for all technologies mixed traffic users among FSA, FSA_RAND, GA-based DSA scheme and TOT_RAND over duration of 24 hours of the day.

during a span of one day. The BLOCKED FSA graph shows GoS for UMTS when different technologies users grab the channel slots in the allocated frequency spectrum slots only by using FSA which is existing regulation. The BLOCKED_TOT_RAND graph shows the GoS for the combined user traffic when there is no regulation and any technology user can grab the channel at random position if it is available. The BLOCKED_FSA_RAND graph is of the randomized channel grabbing FSA where the spectrum is divided among the various technologies but the channel grab is at any random position provided it is vacant. The BLOCKED_GA graph shows the GoS for combined user traffic with GA optimized DSA algorithm which is proposed regulation. It is seen that GA optimized DSA algorithm is always better than all other cases. Also it has approximately up to $55 \%$ improvement in comparison to FSA which is the best performing algorithm amongst the three. If the mean GoS is taken for the given time then a maximum of $80 \%$ improvement is noticed neglecting the cases where DSA gives zero blocking.

\section{Conclusions}

In this study, an attempt is made to analyze the impact of our proposed GA based optimized DSA mechanism for spectrum utilization by comparing GoS for existing
FSA based regulation with proposed mechanism. Four prominent commonly used technologies are used for the simulations which occupy different bandwidths to analyze the impact of our proposed Genetic Algorithm based solution which uses aspects of cognitive radio for improving the overall GoS of a shared cellular spectrum scenario. The simulation results can be utilized to justify the need of regulatory approach in case of liberalized spectrum sharing in the present cellular network spectrum in the preview of cognitive radio. As it can be seen from results in Figures 3 to 7 that GA based algorithm enhances the GoS when compared to the present allocation scheme. The maximum value of improvement in GoS for GSM, CDMA, UMTS, WiMAX and mixed traffic are $72 \%, 72 \%, 30 \%, 15 \%$ and $55 \%$ respectively. The improvement in GoS for GSM, CDMA, UMTS, WiMAX and mixed traffic around mean values are $67 \%$, $95 \%, 60 \%, 70 \%$ and $80 \%$ respectively. This study shows some sort of regulatory based approach within liberalized spectrum sharing concept is required to allow all users to get equal chance of utilizing the spectrum and getting a better GoS for the overall traffic.

\section{References}

[1] M. P. Olivieri, G. Barnett, A. Lackpour, A. Davis, and P. 
Ngo, "A scalable dynamic spectrum allocation system with interference mitigation for teams of spectrally agile software defined radios," New Frontiers in Dynamic Spectrum Access Networks, pp. 170-179, November 2005.

[2] J. Mitola III, "Cognitive radio an integrated agent architecture for software defined radio," Dissertation, Doctor of Technology, Royal Institute of Technology (KTH), Sweden, May 2000.

[3] Paul Burns, "Software defined radio for 3G," Artech House, Inc., 2003.

[4] J. Hwang and H. Yoon, "Dynamic spectrum management policy for cognitive radio: An analysis of implementation feasibility issues," New Frontiers in Dynamic Spectrum Access Networks, 3rd IEEE Symposium, Digital Object Identifier, pp. 1-9, October 2008.

[5] S. Haykin, "Cognitive radio: Brain-Empowered wireless communications," IEEE Journal on Selected Areas in Communications, Vol. 23, No. 2, February 2005.

[6] Bruce A. Fette., editor, "Cognitive radio technology," Elsevier Inc., 2006.

[7] S. Almeida, J. Queijo, and L. M. Correia, "Spatial and temporal traffic distribution models for GSM," Vehicular Technology Conference, Vol. 1, pp. 131-135, September 1999.

[8] A. Y. Zomaya, Senior Member, IEEE, and Michael Wright, "Observations on using genetic-algorithms for channel allocation in mobile computing," IEEE Transactions on Parallel and Distributed Systems, Vol. 13, No. 9, September 2002.

[9] S. S. M. Patra, K. Roy, S. Banerjee, and D. P. Vidyarthi,
"Improved genetic algorithm for channel allocation with channel borrowing in mobile computing," IEEE Transactions on Mobile Computing, Vol. 5, No. 7, July 2006.

[10] M. Melanie, “An introduction to genetic algorithm," MIT press, Cambridge, 1999.

[11] D. Maldonado, B. Le, A. Hugine, T. W. Rondeau, and C. W. Bostian, "Cognitive radio applications to dynamic spectrum allocation: A discussion and an illustrative example," New Frontiers in Dynamic Spectrum Access Networks, First IEEE International Symposium, pp. 597-600, November 2005.

[12] P. Leaves, S. Ghaheri-Niri, R. Tafazolli, L. Christodoulides, T. Sammut, W. Staht, and J. Huschke, "Dynamic spectrum allocation in a multi-radio environment: Concept and algorithm," 3G Mobile Communication Technologies, Second International Conference on (Conference Publication No. 477), pp. 53-57, March, 2001.

[13] J. Zhao, H. T. Zheng, and G. H. Yang, "Distributed coordination in dynamic spectrum allocation networks," New Frontiers in Dynamic Spectrum Access Networks, pp. 259-268, November 2005.

[14] Viswanathan and Thiagarajan, "Telecommunication switching systems and networks," Prentice-Hall, New Delhi, 1992.

[15] D. Thilakawardana, K.Moessner, and R.Tafazolli, "Darwinian approach for dynamic spectrum allocation in next generation systems," IET Communications, Centre for Communication Systems Research, University of Surrey, Guildford, UK, 2008. 